\title{
Civilisations
}

Revue internationale d'anthropologie et de sciences

humaines

66 | 2017

L'alcool rituel et les ethnographes

\section{Bien boire, mal boire. Regards sur l'ivresse des guérisseurs malgaches}

\section{Olivia Legrip-Randriambelo}

\section{(Q) OpenEdition \\ Journals}

Édition électronique

URL : http://journals.openedition.org/civilisations/4281

DOI : 10.4000/civilisations.4281

ISSN : 2032-0442

\section{Éditeur}

Institut de sociologie de l'Université Libre de Bruxelles

\section{Édition imprimée}

Date de publication : 31 août 2017

Pagination : 43-58

ISBN : 978-2-9602017-1-0

ISSN : 0009-8140

Référence électronique

Olivia Legrip-Randriambelo, «Bien boire, mal boire. Regards sur l'ivresse des guérisseurs malgaches », Civilisations [En ligne], 66 | 2017, mis en ligne le 31 août 2020, consulté le 25 février 2021. URL : http:// journals.openedition.org/civilisations/4281; DOI : https://doi.org/10.4000/civilisations.4281 


\title{
Bien boire, mal boire Regards sur l'ivresse des guérisseurs malgaches
}

\author{
Olivia LEGRIP-RANDRIAMBELO
}

Résumé : La consommation de rhums artisanaux, industriels et/ou de bières est un invariant des rituels de soins engagés par les devins-guérisseurs malgaches, qu'ils soient possédés par des esprits ou non. Cet article propose de s'intéresser aux regards que posent les patients sur l'ivresse, et au contraire, sur la résistance à la consommation d'alcool de leur thérapeute. J'aborderai ainsi le "bien boire " avec le cas d'une possédée réputée pour son absence d'ébriété lors de rituels pourtant fortement alcoolisés, et le " mal boire » avec l'exemple d'un devin-guérisseur, buvant démesurément, et dont les patients déprécient alors les compétences. Autrement dit, il sera question, à partir du discours des pratiquants, de la manière dont les compétences sont proportionnelles à la quantité d'alcool ingéré et à la maîtrise de soi. La consommation d'alcool rituel oscille ici entre une gageure de puissance magico-religieuse et une dépendance néfaste au rituel de soin. Ainsi, l'alcool trouve sa place dans le rituel et justifie son aspect magico-religieux mais peut également le conduire à l'échec : en effet, la démesure liée à la consommation d'alcool n'est autorisée que dans la limite de l'activité rituelle, dans la mesure rituelle. L'action rituelle est alors l'unique contexte légitime de son usage.

Mots-clés : anthropologie des religions, alcool rituel, culte ancestral, culte de possession, Madagascar.

\begin{abstract}
The consumption of artisanal or industrial rum and/or beer is an invariant of the rituals of care engaged by the Malagasy healers-diviners, whether possessed by spirits or not. This article proposes to look at the views of patients on drunkenness and on the resistance to alcohol consumption of their therapist. I will discuss the "good drinking» with the case of a possessed woman known for her lack of drunkenness during rituals yet strongly alcoholic, and «bad drinking» with the example of a healer-diviner drinking disproportionately, and whose patients depreciate the skills. In other words, I question, based on practitioners'discourses, of how skills are proportional to the measure of alcohol ingested and self-control. The consumption of ritual alcohol oscillates between a betting of magico-religious power and a detrimental dependence on the ritual of care. Thus, alcohol finds its place in the ritual and justifies its magico-religious aspect, but it can also lead to failure: excesses related to the consumption of alcohol are permitted only within the limit of ritual activity, to the ritual extent. Ritual action is then the only legitimate context for its use.
\end{abstract}

Keywords: anthropology of religions, ritual alcohol, ancestral cult, possession cult, Madagascar. 


\section{Introduction}

Dans la région betsileo, autrefois, « à la mort des souverains de l'Isandra (pays betsileo), les 'gens du sacré' (olom-pady), chargés de veiller sur la dépouille royale et de recueillir les liquides, buvaient du rhum avec leurs mains 'enduites de sanies gluantes'. Cette communion avec les ancêtres n'est possible que dans le cadre des funérailles d'un personnage déjà médiateur entre les divinités et les vivants » (Beaujard 2014 : 149-150). Un manuscrit de la London Missionary Society souligne également la «place d'honneur » du rhum dans ce rituel funéraire. La toilette mortuaire est effectuée avec de l'eau et de l'alcool, plus particulièrement du rhum artisanal parallèlement usité lors des rituels destinés aux ancêtres et « bu en abondance par les participants aux funérailles » (Beaujard 2014 : 150 ; Callet 1908 ; Rahamefy 1997). Philippe Beaujard, dans une perspective ethno-historique, précise :

«l'hydromel (ou un alcool de canne) convient aux rites de passage, mettant en scène une mort symbolique suivie d'une renaissance, qu'il s'agisse d'un enfant, d'une jeune fille (mariage), d'un malade ou du chef de la communauté, rites qui impliquent les ancêtres. [...] Le fait que se mêlent dans les tromba betsimisaraka ${ }^{1}$ esprits de la vie et mânes des ancêtres contribue sans doute pour une part à expliquer la présence conjointe du miel et de l'hydromel dans les rituels. Celle-ci tient aussi plus fondamentalement à la structure même des rituels, qui mettent en scène une mort symbolique et une renaissance. Celle-ci est le résultat d'une fécondation ou d'une création induite par des substances de vie associées aux esprits de la nature (miel), aux ancêtres (alcool) et aux divinités suprêmes (Zañahary, Andriamañitra) (sang) » (2014:151-153).

Je propose ici de m'intéresser, à partir d'une recherche doctorale menée dans cette région sur les pratiques religieuses de guérison, aux regards que posent les patients sur l'ivresse, ou au contraire, sur la résistance à la consommation d'alcool de leur thérapeute. Tous les devins-guérisseurs rencontrés sur ce terrain ethnographique boivent, dans le contexte rituel, de l'alcool en plus ou moins grande quantité - qu'ils trempent les lèvres dans la boisson par respect des codes rituels ou qu'ils apprécient de grandes et fréquentes gorgées ${ }^{2}$. Parmi tous mes interlocuteurs guérisseurs, mon choix pour exemplifier ce texte s'est porté sur deux d'entre eux, Sainte et RaJaona ${ }^{3}$, car d'une part, ils consomment de l'alcool en grande quantité ${ }^{4}$, dans le temps rituel pour la première et également en dehors de ce temps sacré pour le second, et d'autre part (et surtout), leurs patients ont choisi d'évoquer avec moi, en présence ou non de leur thérapeute, le rapport de ce dernier à l'alcool - qu'ils le jugent religio-thérapeutique ou non.

1 Culte de possession de la côte est de Madagascar.

2 Ce point est à élargir à l'ensemble de Madagascar où les recherches portant sur le religieux font toutes mention de l'utilisation de l'alcool rituel. Voir particulièrement Rakotomalala et al. 2001 ; Blanchy et al. 2006 (pour les Hautes Terres centrales) ; ou encore Estrade 1985 ; Austnaberg 2008 ; etc.

3 Compte tenu des discours portés sur sa pratique par certains de ses patients, les noms du devin-guérisseur et de son épouse ont été modifiés.

4 La quantité mentionnée ici ne préjuge pas d'une mesure éthylique mais estime la consommation d'alcool en comparaison avec d'autres consommations alcooliques relevées dans d'autres temps d'observation ethnographique menés chez plus d'une dizaine de devins-guérisseurs de la région betsileo. 
Après avoir posé un cadre sur le lien entre l'alcool et la démarche ethnographique, j'aborderai ainsi le "boire » à partir de ces deux cas ethnographiques observés à Fianarantsoa, capitale de la région betsileo au sud des Hautes Terres centrales de Madagascar. Le premier est celui du « bien boire » avec Sainte, une guérisseuse possédée réputée pour son absence de démesure lors de rituels pourtant fortement alcoolisés. Et le second évoque le « mal boire » avec l'exemple de RaJaona, un devin-guérisseur (non possédé rituellement) buvant démesurément, et dont les patients déprécient alors les compétences. Enfin, il sera nécessaire de penser plus généralement comment la question de la mesure et de la démesure de l'alcool rituel oscille entre une gageure de puissance magico-religieuse et une dépendance néfaste au rituel de soin.

Le propos de cet article fait écho à un proverbe malgache très populaire : «ny Betsileo tsy mamo fa ny toaka no mahery », littéralement : " les Betsileo ne sont jamais ivres, mais c'est le rhum qui est trop fort ». Si cette expression populaire est communément utilisée dans le contexte festif, souvent en fin des festivités profanes ou de celles périphériques aux activités sacrées (cf. Obadia 2009, entre autres et dans d'autres contextes ethnographiques), elle peut également être mentionnée par les devinsguérisseurs lors de leurs activités rituelles alcoolisées, par leurs patients partageant leur verre, ou par ceux commentant le rapport de leur thérapeute à l'alcool rituel, alors pensé comme un alcool surconsommé sous couvert de religiosité. Même si je me concentrerai dans ce texte uniquement sur la consommation rituelle, et non sur la consommation récréative ou pathologique, je renvoie sur ce point au travail sur les expressions du boire de Véronique Nahoum-Grappe (1989).

\section{Boissons fermentées et démarche ethnologique}

Boissons et libations organisent et conditionnent la (dé)mesure rituelle de la consommation d'alcool. L'usage de l'alcool et d'une possible surconsommation de ce dernier par les devins-guérisseurs en fait un « objet liminal » (Givre 2006 : 83-85) et ambivalent enchâssé dans le rituel et le monde séculier. Les boissons et le " boire » possèdent des fonctions symboliques et sociales : l'alcool est utilisé en libation et consommé en quantité variable comme substance nécessaire à l'efficacité rituelle. Quant au « boire », il signifie un agir codifié par le rituel, un garde-fou de la pratique et de la consommation. L'alcool est envisagé ici à la fois comme objet et comme pratique rituelle, une sociabilité rituelle qui se détache des études sur l'alcool pathologique (Douglas 1987 ; Fabre-Vassas 1989 ; Fainzang 1995). Autrement dit, pour ce qui concerne « l'ethnographie des manières de boire [...], cette démarche [...] se démarque de la sociologie et de l'épidémiologie de l'alcoolisme » (Castelain 1996 : 181).

Les boissons fermentées sont, dans le contexte rituel, un outil de mesure des compétences magico-religieuses du buveur, autant dans la démesure de leur consommation que dans la gestion de la sobriété. Dans le discours des patients, les compétences sont proportionnelles à la quantité d'alcool ingéré et à la maîtrise de soi. Les devins-guérisseurs expérimentent leurs limites face à un abus de consommation d'alcool. Ils se trouvent ainsi dans un jeu de bascule permanent entre mesure et démesure, qui crée la maîtrise, ou l'absence de maîtrise, justifiant leurs capacités rituelles. L'alcool trouve sa place dans le rituel mais peut également le conduire à l'échec : le dépassement n'est autorisé que dans la limite de l'activité rituelle, dans la mesure rituelle. Dans une étude des transes de possession collective malgaches (tromba), Paul Ottino remarque 
que lors des appels aux esprits, « il n'est pas rare que les possédés, étourdis de fatigue par l'alcool [...] s'effondrent, épuisés » avant que l'esprit ne se manifeste (1965:86). La démesure de la consommation d'alcool fait partie intégrante de la temporalité rituelle et de ses codes.

Dans cette étude ethnographique, il s'agit de définir les fonctions rituelles/symboliques et les usages des libations et de l'absorption d'alcool (hydromel, rhum, bière, etc. qu'ils soient artisanaux ou industriels ${ }^{5}$ ). Dans un second temps, il est nécessaire de saisir la démesure de leur utilisation, car la réalité du buveur dépasse parfois les codes de consommation réelle et symbolique imposés par le rituel. En somme, il s'agit de prendre en compte l'alcool dans un espace et un temps rituels qui lui donnent tout son sens (Fabre-Vassas 1989). Il est un élément du rituel mais la quantité consommée n'est pas imposée par les esprits et/ou les ancêtres. A Madagascar, dans les rituels organisés par les devins-guérisseurs (ombiasa, possédés tromba, etc.), l'alcool, très souvent du rhum artisanal, est une substance obligatoire ; néanmoins sa quantité varie et peut amener à un excès éthylique. Le consommateur boit à la bouteille ou au verre, seul ou avec l'assemblée, en son nom ou au nom des esprits qui le possèdent. La fréquence de consommation d'alcool rythme les étapes du rituel (de la présentation du/des esprits à son/leur départ, en passant par les demandes, les soins ou les sacrifices).

\section{Sainte et l'alcool performatif}

\section{Quand l'esprit boit à travers la possédée}

Le terme tromba, comme dans la grande majorité des cultes de possession, désigne l'esprit qui possède, le possédé, le rituel et le type de possession. Il peut être rapproché des rituels décrits dans les travaux menés en Inde par Gilles Tarabout comme « l'un des modes de contact entre dieux (ou esprits) et humains, et non comme état de conscience modifié (et a fortiori comme trouble mental), [il] est donc partie intégrante des représentations et des pratiques socioreligieuses » (1999:17). Les spécificités des cultes du nord-ouest de Madagascar, également rapportés en Imerina (région de Antananarivo), où les possédés sont habités ou visités par d'anciens rois locaux, ont été traitées dans de nombreux travaux anthropologiques ${ }^{6}$. Il en est question ici uniquement dans leur aspect religio-thérapeutique et leurs implications dans l'organisation sociale : les esprits peuvent soigner eux-mêmes par le biais de la possession, sans même que le guérisseur ait la nécessité d'acquérir un savoir phytothérapique personnel. L'esprit soigne et le guérisseur n'est que le canal de communication par lequel il s'exprime. En somme, le guérisseur ne soigne pas lui-même, à l'instar, de Sainte.

Sainte est née en 1989 et réside à Fianarantsoa. A l'âge de 7 ans, elle a fait face à un invariant de l'acquisition des aptitudes de l'activité médiumnique : une grave maladie, qui a été le phénomène déclencheur et révélateur de la présence d'esprits. Après un an de va-et-vient entre un devin-guérisseur et l'hôpital, des « choses lui

5 Sur la consommation et les diverses représentations des alcools locaux/artisanaux et importés/ manufacturés, voir les travaux menés en Chine par Magnus Fiskesjö (2010).

6 Comme, entre autres, ceux de Gérard Althabe (2000, 2002 [1969]), Jean-Marie Estrade (1985), Michèle Fiéloux et Jacques Lombard (1991, 1995), Lesley Sharp (1993), Hilde Nielssen (2012). Pour ces cultes dans le nord des Hautes Terres, voir les travaux de Paul Ottino (1965), Sophie Blanchy et Rahajesy Andriamampianina (2001).. 
sont apparues $\gg^{7}$ (nisehoan-javatra). Suite à cette révélation, le vocabulaire utilisé par Sainte pour évoquer la présence des esprits est marqué par deux idées : tout d'abord celle de la possession, « d'être placé » (mipetraka), mais aussi celle de l'incorporation, d'effectuer un mouvement du haut vers le bas, de "faire descendre " (mampidiña). Si le mouvement est explicite, les esprits en revanche sont toujours mentionnés par un vocabulaire de l'indéterminé : zavatra désigne une chose ou un esprit (au sens très vague); ou raha, qui désigne une substance.

Elle s'est ensuite rendue à Antananarivo, où vit sa tante, elle aussi possédée tromba, pour être initiée. Son chemin initiatique s'est poursuivi jusqu'en région sakalava, fief des esprits tromba, sur la côte nord-ouest pour un bain de mer rituel. A son retour en région betsileo, Sainte vivait et soignait dans un petit village de la campagne environnante de Fianarantsoa, avant de venir en ville poursuivre ses études secondaires dans une école catholique. Elle prodiguait alors les soins uniquement les jours chômés : les mercredis après-midi et samedis. Au fil du temps, les sœurs catholiques ont découvert ses activités extrascolaires et ont refusé de l'inscrire en classe supérieure, justifiant leur refus en invoquant la possession de Sainte par le Diable. Cet évènement marque le début de ses activités à temps plein, à l'âge de 17 ans. Après avoir soigné un jeune homme ensorcelé risquant une mort imminente, elle l'a épousé et pratique depuis, dans une salle dédiée aux soins au rez-de-chaussée de la maison de sa belle-famille, à Fianarantsoa.

\section{Les conduites alcooliques «masculines » d'une jeune fille}

Sainte est une jeune fille timide et apprêtée, mais lorsqu'elle est possédée par des esprits royaux masculins, elle se mue en ces hommes et assume des pratiques socialement transgressives pour une jeune fille dans le contexte malgache, comme fumer et boire de l'alcool sans discontinuité lors des consultations. Dès lors, comme le suggère Gérard Althabe, « le sexe du possédé n'a plus d'importance » (2002 [1969] : 99), le changement d'un corps féminin frêle et discret à des postures corporelles masculines et royales émerge lors de l'incorporation. Les rois se manifestent à travers la voix masculinisée de la jeune femme, qui couplée à des postures significatives accentuent leur statut et leur prestance. Elle acquiert la personnalité de ces esprits virilisés symboliquement par l'histoire et le rituel, au profit de la sienne. La « maîtrise des comportements ostentatoires propres à chaque esprit atteste cette aptitude dont doivent faire preuve tous les initiés », comme l'a remarqué Bertrand Hell (2008 : 11) chez les tromba malgachophones de Mayotte, dont la consommation d'alcool rituel est visible et performative. La posture, les offrandes alcooliques consommées lors de la transe, les tics verbaux et l'accent varient d'un esprit à l'autre permettant ainsi à l'assistance de savoir qui se manifeste à travers Sainte.

7 Les propos cités dans cet article sont tous des traductions d'extraits d'entretiens ou de discours recueillis entre 2010 et 2013 dans la communauté urbaine de Fianarantsoa.. 


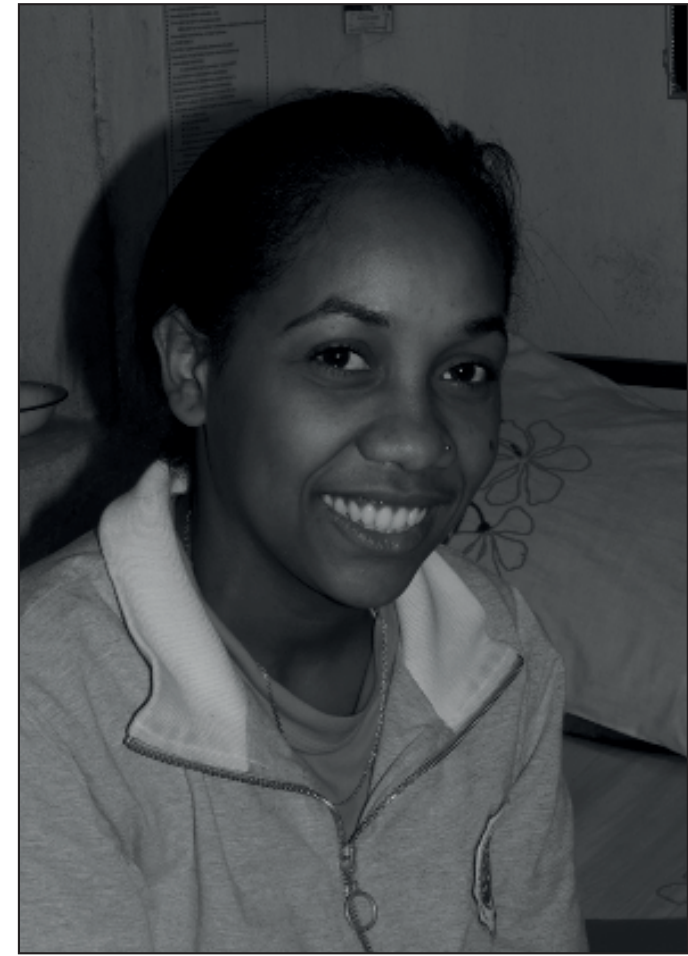

Figure 1 : Sainte avant la transe de possession (C) Olivia Legrip, 2013



Figure 2 : Sainte possédée, whisky en main (C) Olivia Legrip, 2013

A chaque séance, le consultant doit se munir de deux bouteilles de $75 \mathrm{cl}$ de bière, de deux bougies, d'un paquet de cigarettes et d'un paquet de bâtonnets d'encens. A cela s'ajoutent des boissons supplémentaires selon l'esprit en présence et ses obligations rituelles. Un membre de la famille de Sainte court acheter avec l'argent du patient des sodas pour les esprits qui ne consomment pas d'alcool, ou du rhum ou du whisky pour ceux qui les préfèrent. Certains esprits ont une interdiction de consommation d'alcool : le plus souvent, il s'agit des esprits féminins. Dans le cas de Sainte, elle est face à l'ambiguïté d'une ivresse divine nécessaire couplée à l'interdiction d'alcool pour certains esprits, et plus encore, d'une interdiction sociale pour une jeune femme. C'est bien cette contradiction dans le rapport entre la jeunesse, le genre féminin et la conduite alcoolique qui fait de ce cas de possession un élément ethnographique et analytique intéressant.

Finalement, Sainte commence par haleter, puis elle pousse des râles tandis que ses mains font pression sur sa poitrine. Après la présentation du tromba, les consultations se déroulent sous la forme d'un dialogue entre l'esprit et le patient ponctué de gorgées d'alcool partagées, puis viennent le diagnostic et les prescriptions. Ici, comme dans beaucoup de contextes de possession imposant une grande consommation d'alcool rituel, dont la Mongolie par exemple, le possédé « doit non seulement offrir de l'alcool distillé à ses esprits auxiliaires, mais lui-même en consommer» (Lacaze 2004 : 46).

Sainte est assise devant l'autel, les consultants sont sur des nattes perpendiculairement à elle. Son époux (et assistant) est accroupi avec les patients. Les tromba réclament en permanence qu' on allume leurs cigarettes et qu' on leur serve à boire. Occasionnellement, ils proposent de partager leur boisson avec l'assemblée. La séance rituelle n'est possible que si tous les individus présents y participent en suivant ce que Paul Ottino appelle des 
« conduites collectives régulières » $(1965: 84)$ connues de tous ou exposées en début de séance, dont la consommation collective de l'alcool rituel. L'esprit s'échauffe en cas de refus de boire quel qu'en soit le motif (la quantité, l'heure de la journée, etc.). A l'issue de la séance, et une fois le traitement terminé ou la protection mise en place, les tromba imposent des interdits ( $f a d y)$ et menacent les consultants, par exemple : les esprits briseront une bouteille de bière sur la tête de celui qui dira du mal d'eux. Là encore, une référence est faite à l'alcool.

«Donne-moi mon Brighton et reprends ta bière !»

Un des esprits princiers qui possède Sainte se nomme Rakotomaditra : c'est une figure réputée à l'échelle nationale (cf. entre autres Rakotomalala et al. 2001 : 329, 449). Il affectionne particulièrement l'alcool. Chez Sainte, il réclame systématiquement du Brighton, un whisky manufacturé. Lors d'une consultation, la jeune femme possédée par l'esprit de Rakotomaditra offre du whisky au malade en traitement pour une douleur à l'œil. Après qu'un long rituel de divination et de guérison ait été performé, le malade annonce ne plus avoir mal, tout en buvant une rasade au goulot de la bouteille de whisky que lui a tendu la possédée. L'esprit de Rakotomaditra, satisfait d'apprendre que le malade sent déjà une amélioration, lui tend la bouteille de THB, une bière industrielle de fabrication malgache, et lui lance : « Ha ! Bien! Et puis merde, donne-moi mon Brighton et reprends ta bière ! ». Bière et alcool fort se partagent et se croisent dans les rituels mais ils ont des rôles et des attributions différents. Ils permettent notamment de distinguer les esprits selon leurs préférences en matière de boissons. A la suite de Philippe Beaujard et dans une continuité ethnographique, nous remarquons que :

« plus encore que l'hydromel, toutefois, c'est ici la bière qui retient l'attention dans une anthropologie comparatiste. Liée au monde des morts, elle jouait un rôle dans les cérémonies funéraires, avec en arrière-plan la même symbolique que pour l'hydromel ou le rhum à Madagascar » (2014:159).

Dans un contexte tout à fait différent, la bière a aussi été analysée comme une « boisson des morts dans l'au-delà [...] la bière est naturellement appelée à jouer un rôle important dans la vie quotidienne lors des cérémonies funéraires » (Hell 1991 : 110, 118). Cette étude menée en Allemagne montre la valeur heuristique de la symbolique accolée à l'alcool. Bertrand Hell poursuit : " c'est à partir de cette mise en relation entre la bière et les morts que peut s'établir le lien plus étroit entre pratiques inscrites dans le mythe et croyances populaires » (Ibid.). Ainsi, et en nous situant à un niveau plus général sur la symbolique et les représentations de l'alcool rituel, le distillé et le fermenté sont modifiés " sans perdition de substance » et dans une " enceinte close sur elle-même » (Lévi-Strauss 1964 : 24), « or, entre fermentation et décomposition se situe une limite critique qui, sans l'action de l'homme (ou de la nature) sur la matière, ferait basculer le sublimé vers le toxique » (Bianquis 2012 : 20-21). L'alcool rituel pour le cas malgache est vertueux et chargé d'un entre-deux performatif qui oscille entre l'usage qu'en font les Hommes et les officiants du culte, et l'offrande faite aux entités comme ouverture d'un canal de communication entre eux. Néanmoins, l'alcool peut se muer en breuvage non vertueux exempt de ses qualités rituelles et devenir une boisson toxique pour le buveur.

Outre une réputation englobant à la fois le lieu, le rituel et les guérisons, la possession 
tromba de Sainte est également la source principale du revenu familial. L'originalité de cette pratique rare dans la région betsileo, associée au jeune âge de la guérisseuse, est la pierre angulaire des activités de cette salle de consultation construite autour de la figure de guérisseuse possédée de Sainte. Il est courant d'entendre les membres de sa bellefamille se vanter de l'étonnement des patients guéris face aux compétences de Sainte : « Ha ! Cette petite fille peut faire ça !», ce qui implique la guérison bien sûr, mais aussi sa résistance certaine à l'alcool.

Le fait que Sainte soit une jeune femme mince et discrète est une valeur ajoutée à sa réputation ; sa transformation en un roi sakalava insatiable buveur n'en sera que plus impressionnante. L'ivresse-possession, pour reprendre l'expression formulée par Eric Jolly dans un tout autre contexte, est alors spectaculaire (2004 : 220). Il arrive que cette vertu soit délibérément sollicitée et mise en scène dans un rite (Fabre-Vassas 1989). L'étonnement des patients renvoie aussi à son jeune âge, comme dans le cas de Felasoa à Madagascar, âgé de 25 ans, et qui est jugé comme un " enfant connaisseur » : ajaja mahay raha (Chemillier et al. 2007 : 14). Sainte a, par ailleurs, temporairement arrêté sa consommation d'alcool pendant sa grossesse. Lorsque les esprits ont su qu'elle portait un enfant, ils lui ont tous stipulé qu'elle devait s'abstenir d'ingérer toute boisson alcoolisée.

Le parler et l'accent sakalava, les murmures et les digressions des esprits ivres nécessitent l'intervention du mari de Sainte qui traduit puis transcrit sur papier ses paroles parfois balbutiantes, jouant ainsi le rôle d'intermédiaire, d'assistant. Sainte exporte le modèle sakalava en région Betsileo et propose des rituels de transe dynamiques et théâtralisés dans lesquels la démesure alcoolique est un acteur principal, ainsi elle joue de l'attente d'un exotisme sakalava recherché par certains de ses patients betsileo (Legrip-Randriambelo 2014 : 96). La présence rituelle « d'êtres du lointain » qui appartiennent à «l'univers exotique » interne ou national (Petit 1997 : 187) participe de la particularité régionale de Sainte.

En effet, l'appellation sakalava - région nord-ouest de Madagascar - «tromba » peut être utilisée et réceptionnée dans le contexte betsileo par une référence faite à deux cultes de possession régionaux historiques que sont le bilo et le salamanga, rapprochés, décrits et observés par le missionnaire ethnographe Henri Dubois (1938) ou encore par Jacques Faublée (1946 : 118). Henri Dubois définit ces deux termes au début du 20 siècle : le salamanga est « individuel et volontaire », tandis que le bilo est " épidémique et contagieux » (1938: 1065), et il voit le tromba sakalava comme un « succédané » ou un « cousin » (ibid. : 1077-1078) du salamanga betsileo. Paul Ottino décrit le tromba et le bilo qui, pour lui, " se déroulent dans une atmosphère comparable » dont la présence d'alcool et la possession sont deux des éléments principaux (1965 : 85). Bien que ces cultes aient aujourd'hui disparu en tant que transe collective, les termes salamanga et bilo sont encore parfois employés par des devins-guérisseurs ou leurs épouses pour qualifier des faits de possession. Dès lors, en région betsileo, entre les esprits ancestraux et les esprits qui possèdent (tromba), la question d'une anomalie régionale se pose : les possessions tromba n'ont été observées que sur les côtes de l'île et au nord des Hautes Terres dans la région de Antananarivo, mais sont a priori inexistantes dans la région betsileo (Estrade 1985 : 188 ; Beaujard 1995 : 194).

Cet exotisme peut passer, pour certains autres guérisseurs en concurrence avec Sainte, 
pour du charlatanisme, pour un savant jeu d'actrice renvoyant à des pratiques masquées de sorcellerie ; essentiellement basé sur le fait qu'une si jeune femme qui boit autant d'alcool ne peut pas être vertueuse.

Retournons au déroulement du rituel chez Sainte où toutes les personnes présentes boivent une gorgée de toaka mena (« rhum rouge », souvent manufacturé, en opposition aux alcools blancs, jus de canne fermenté ou rhum artisanaux), qui est alors appelé barisa, le terme sakalava désignant le rhum utilisé dans le contexte des transes de possession dans les Hautes Terres. Avec l'utilisation d'un vocable sakalava en région betsileo, c'est bien une référence à la possession tromba, particularité de la guérisseuse qui est mise en avant. La dénomination de l'alcool bu rituellement est ici mobilisée comme une définition de la pratique rituelle elle-même. De plus, le barisa, défini par Jean-Marie Estrade comme du « miel porté par la cuisson à la couleur rouge, [qui] a le pouvoir de faire naître la transe » (1985 : 129-130), renvoie explicitement à la possession tromba. Il peut également être rapproché des esprits de la terre et des ancêtres. Le terme barisa, lourd de sens et de représentations religio-thérapeutiques, désigne encore une « sorte de consécration d'un malade qui assume la possession et au cours de laquelle on boit le barisa », ce qui évoque, encore une fois, la consommation d'alcool rituel (Estrade 1985 : 129-130 ; Beaujard 2014 : 154).

Dans ce contexte, Sainte boit sans pourtant accéder à l'ivresse, mais son époux et assistant rituel essaye de la ménager (il dit s'inquiéter de sa consommation d'alcool et de tabac). Alors que le soin mentionné ci-dessus est terminé, et la nuit déjà bien avancée, l'esprit de Rakotomaditra boit et plaisante encore. Hans Austnaberg note que plus la transe de possession s'installe chez les possédés, plus la quantité d'alcool bu augmente (2008 : 159). L'époux de la possédée décèle des signes de fatigue chez elle. En effet, après une séance de transe, les possédés sont fatigués, mais ne ressentent pas les effets de l'alcool ingurgité durant la cérémonie (Ibid.). L'époux de Sainte commence à contrer les propos de l'esprit, il refuse désormais de boire aux bouteilles qu'il lui tend et l'a excédé en chuchotant qu'il souhaitait que Sainte s'arrête pour ce jour. Tandis que l'esprit est occupé à boire, le mari dit au malade de s'asseoir plus loin de la possédée, afin d'amorcer son départ, et surtout parce que Rakotomaditra, une fois ivre, « devient fou la nuit. Si vous restez, vous êtes là jusqu'à demain matin... », dit-il.

C'est à ce moment, en fin de séance, que l'esprit de Rakotomaditra s'adresse à moi, me tendant une bouteille et une cigarette. Il choisit ce temps liminaire, entre sacré et profane, pour aborder ma recherche. Il annonce être ivre et de ce fait, m'avoir livré une partie de ses secrets, ce qui selon ses propos, nous lie désormais. Autrement dit, il est possible de percevoir là une acceptation de présence sur le terrain, une place signifiée et signifiante attribuée pour le temps rituel et un moyen de collecte de données ethnographiques, tout ceci permis en partie grâce à l'alcool que nous avons partagé rituellement.

\section{RaJaona et l'alcool dépréciatif}

\section{L'alcool rituel d'un devin-guérisseur désorcelleur}

RaJaona vit avec sa femme, Odette, à l'extrémité nord de la communauté urbaine de Fianarantsoa. Il se définit comme un mpitaiza (littéralement, « celui-qui-prend-soin », « celui-qui-nourrit ») : ce terme évoque l'idée du médecin de famille qui suivra ses 
membres sur plusieurs décennies.

A l'âge de 20 ans, sans savoir ce qui l'y poussait, il a couru jusqu'à la rivière voisine et a sauté dans l'eau : " on aurait dit que j'étais fou !». Il dit avoir agi comme s'il était poursuivi. Cet épisode n'était pas le premier signe envoyé par les esprits ancestraux dont le guérisseur sent la présence depuis sa dixième année, mais il fut l'élément déclencheur de sa fonction actuelle et de la compréhension de ses ressentis d'enfant. Il raconte son immersion aquatique qui dura sept jours comme un moment de solitude pourtant accompagné par des lolondrano (« esprits-des-eaux »). Le don qu'il qualifie, comme la majorité de mes interlocuteurs, de «naturel» (en français) lui a été transmis par son père décédé, lui-même guérisseur en contact avec les entités aquatiques et leurs ancêtres familiaux. Ce matin-là, à l'arrivée de Georgelin, un homme d'une quarantaine d'années, Odette, l'épouse de RaJaona, tient particulièrement à me le présenter :

«Lui, il vient souvent chez nous ! A chaque fois qu'il a mal, il vient et c'est toujours ça [les sisika ${ }^{8}$, sujet que nous abordions à ce moment] qu'il [RaJaona] enlève de son corps. Moi-même, je me demande d'où sortent ces trucs ! Je suis vraiment troublée ! Comment ces trucs-là sont entrés !? Lui, c'est notre taiza [littéralement, « celui dont on prend soin »] depuis longtemps ».

L'auscultation à l'aide du miroir divinatoire débute. Georgelin souffre de maux de dents et de dos. RaJaona prépare ses outils rituels en les plongeant dans de l'eau sacralisée, appelle les ancêtres, offre du toaka gasy (rhum artisanal, « rhum malgache ») sur l'autel, puis en ingère une rasade, se gargarise et crache le contenu de sa bouche. Il dit que le toaka fait partie du rituel mais il prouve également ainsi qu'il ne dissimule aucun morceau de bois dans sa gorge, son palais ou ses joues. RaJaona ôte à Georgelin, par aspiration buccale, deux sisika sous forme de bois, le premier de la mâchoire et le second de l'omoplate droite. Odette commente fièrement, comme à chaque fois : « tu vois ça ? Il s'est vraiment rincé la bouche avant et il n'y avait pas de bois ! ». Dans ce contexte le rhum artisanal a également fonction de purifiant et de preuve de l'absence de sisika dans la bouche du guérisseur. Il est utilisé aux marges du rituel. Georgelin est resté dans la salle de soin pendant les consultations suivantes. Il a manifesté une envie de me relater son parcours de soins et de me parler de RaJaona après la matinée de travail du devin-guérisseur - nous y viendrons plus loin.

\section{Boire pour le rituel ou s'arranger avec le rituel pour boire?}

Isabelle Bianquis distingue le « boire ensemble » du « boire sérieusement » $(2012$ : $172,180)$; en ce sens, RaJaona met en œuvre un système rituel d'accueil des malades et de libations lui permettant d'ajuster la frontière entre ces deux manières de boire.

RaJaona subit, puisqu'il ne les maîtrise ni ne les provoque, ces bilo depuis 1976. Les entités qui se manifestent alors sont ses ancêtres familiaux qui l'aident quotidiennement lors des soins et qui durant le temps du bilo évoquent leur mécontentement. Le guérisseur

8 Les sisika (« qu'on pousse »; « qu'on insère entre des choses ») sont des actes sorcellaires courants dans les Hautes-Terres et suspectés dès l'apparition de « tout gonflement [qui] évoque l'insertion sous la peau d'un corps étranger » (Blanchy 2006 : 279). Les sisika sont faits de petits morceaux de bois provenant d'un cercueil ou d'une planche de transport mortuaire placés sur le chemin emprunté par la personne visée qui, en l'enjambant, incorporera l'ensorcellement. Autrement dit, il s'agit d'ensorcellement souscutané sous forme de bouts de bois. 
doit, de fait, les sustenter et les célébrer, souvent durant plusieurs jours. Les motifs de l'apparition désordonnée des ancêtres sont majoritairement liés à la trop importante consommation de rhum artisanal (toaka gasy) par RaJaona en dehors du contexte rituel, ou encore lorsque ce dernier se rend dans une église chrétienne où les cantiques les indisposent. Ce fut le cas lors d'une transe bruyante et incontrôlable de RaJaona au mariage de son jeune frère où les cantiques catholiques ont rendu furieux un des esprits avec lesquels il communique. Son épouse commente l'arrivée du bilo: « il s'est mis à trembler et je l'ai tout de suite amené dehors, dans les bois. Il n'a pas la même façon de vivre que tout le monde... On est restés dans les bois jusqu'à la fin du mariage ». Dès l'arrivée des entités en RaJaona, le guérisseur se trouve dans l'incapacité de se nourrir durant trois journées en guise de punition ancestrale à l'encontre de ces pratiques jugées néfastes à son (et à leur) égard par les esprits. Son épouse ajoute que ce phénomène se répète environ tous les trois mois.

Le fort penchant de RaJaona pour la boisson fait souvent l'objet de réprimandes de la part de ses clients, y compris parmi les plus fidèles et les plus convaincus de son savoirfaire religio-thérapeutique. Si un de ses patients ne le rémunère pas contre ses services ou le dédommage en nature (don d'un poulet, d'un sac de riz, évidemment de rhum, etc.), les ancêtres estimeront que le contrat d'honneur stipulé en début de consultation n'est pas rempli et la maladie ne pourra pas être soignée ; RaJaona négocie alors avec eux le plus souvent une topette de rhum artisanal, dont le prix est accessible.

La méfiance ou la médisance que subit RaJaona de la part de certains de ses patients tient de la crainte de ne pas voir un rituel, biaisé par l'alcool, aboutir à la guérison. Cette crainte inscrit par ailleurs la consommation d'alcool fort (toaka) dans les discours et représentations populaires. Ces références à un alcool fort apparaissent dans l'expression betsileo déjà mentionnée : «ny Betsileo tsy mamo fa ny toaka no mahery », (« les Betsileo ne sont jamais ivres, mais c'est le rhum qui est trop fort »). Parallèlement, l'alcool fort peut aussi discréditer le rituel et rendre fou et/ou malade le buveur, comme cela apparaît dans le célèbre poème «L'ABC de l'alcool » («Abidin'ny toaka ») de Rajaonary datant de 1880 et cité par Claire Riffard (2008:87) :
Adala ny olon'izay doro-dava, Agebok'ireny ny vola ka lany, Ataony ao am-body barika, ka rava:
Areti-mandoza no vokatr' 'izany.
Est fou celui qui s'enivre tout le temps et de son argent ne fait qu'une bouchée, qui met ses avoirs au fond d'une barrique : il récolte des maladies dangereuses.

Ce poème a été édité avec le soutien de la Croix Bleue Malgache (Vokovoko Manga), une organisation protestante prônant l'interdiction de consommation d'alcools et de tabac. Les patients chrétiens qui consultent les guérisseurs sont souvent rassurés par des pratiques syncrétiques ou combinatoires, ce que ne propose pas RaJaona, qui peut alors être exposé aux jugements moraux chrétiens. 


\section{Essai d'analyse sur les alcoolisations rituelles}

Les abus supposés et/ou observés du guérisseur

Suivant ses compétences en matière de désorcellement, tout devin-guérisseur est virtuellement un sorcier. Comme le souligne Lionel Obadia, " les forces de l'antisorcellerie sont nécessairement de même nature que celles de la sorcellerie. Pour combattre les sorciers, il faut soi-même 'en être' un peu » (2005 : 25) ${ }^{9}$. Selon le contexte, les praticiens déploient une palette de leurs aptitudes magico-sorcellaires adaptées à un moment donné, dans une situation donnée. Ils n'utilisent pas toutes leurs connaissances simultanément. Leur rôle d'intermédiaire entre les deux mondes, visible et invisible, va du diagnostic au soin en passant par les désignations des causalités des maux (définis comme sorcellaires ou non), et nécessite alors diverses compétences thérapeutique, magico-religieuse, etc. souvent associées à une grande consommation de rhum artisanal, ou d'un autre breuvage alcoolisé, en contexte rituel.

RaJaona est l'objet de nombreuses rumeurs pourtant contrebalancées par sa réputation de guérisseur compétent. Nombre de ses patients lui reprochent sa grande consommation d'alcool pendant et en dehors des rituels. « Il boit trop ! Plus que trop !» me fait remarquer l'un d'eux au sortir d'une consultation pourtant jugée efficace. Dans ce cas ethnographique, comme dans celui bien différent recueilli en France par JeanPierre Castelain auprès des dockers du Havre, pour ces acteurs à la relation socialement établie à l'alcool : « l'alcoolisme comme maladie n'avait aucun sens » (1996: 186). Parallèlement et en conséquence de son alcoolisme, on reproche à RaJaona des pratiques sorcellaires. Plusieurs des malades rencontrés aux abords de la salle de consultation ont avancé l'idée que le devin-guérisseur conservait les bois, symboles de l'attaque sorcellaire (sisika), qu'il a retirés du corps des ensorcelés et gardés au lieu de les détruire. Lorsque les malades demandent où sont les morceaux de bois extraits, Odette, l'épouse du guérisseur, coupe court à la conversation et dit : «Dada [《 papa»] les a rangés ». Ainsi, il est suspecté de les réutiliser afin de contraindre les malades à revenir, par une manipulation sorcellaire. Les malades évoquent la vénalité du guérisseur, voyant par là un moyen d'accumuler les consultations rémunérées et abreuvées. Un de ses patients s'est ainsi étonné de la longue file d'attente qui se constitue parfois dans la cour de la maison.

Lorsque la matinée de travail de RaJaona touche à sa fin, je quitte son domicile en compagnie de Georgelin. Ce dernier explique comment il interfère dans les rituels alcoolisés de RaJaona. Il le paye en argent mais refuse de lui apporter du toaka (« rhum artisanal») car une trop grande consommation d'alcool risquerait, selon lui, d'empêcher l'extraction du mal : « s'il boit trop, les choses qui devraient sortir vont rester $\gg$, redoute-t-il.

Ces remarques sont autant d'indices empiriques de la puissance accordée à RaJaona, bien qu'elles révèlent un aspect négatif de ses pratiques rituelles dû à sa démesure alcoolique. Les patients estiment être soignés par un guérisseur efficace et continuent de le consulter malgré son alcoolisme notoire et la commercialisation de son activité. 
Autrement dit, dans le discours des pratiquants, les compétences sont proportionnelles à la quantité d'alcool ingéré et à la maîtrise de soi.

\section{Réponses aux invitations à boire}

Les refus aux invitations à boire par les accompagnateurs des malades, avec comme prétexte une justification établie selon les règles de la morale chrétienne (ou les sources que l'Église produit, dont le poème l' «ABC de l'alcool»), l'heure matinale, la quantité à ingérer, ou encore la fréquence de consommation au cours du rituel, ne sont pas acceptables pour le guérisseur ni pour les esprits partageant leur alcool. En effet, cette obligation est nécessaire à l'efficience du rituel pour les malades.

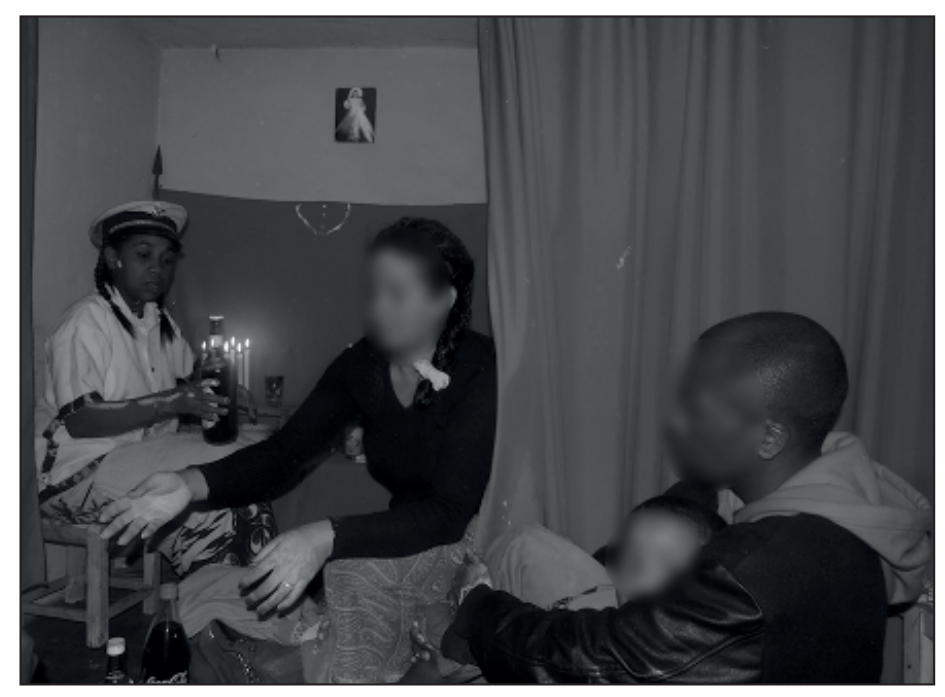

Figure 3 : Circulation de main en main de bouteilles de bière et d'une tasse de rhum chez Sainte (C) Olivia Legrip, 2013

De l'usage rituel à l'usage cérémoniel de l'alcool, pour les esprits autant que pour l'intégration des divers acteurs du rituel, y compris les acteurs silencieux mais néanmoins partie prenante (les accompagnateurs des malades, l'ethnographe, etc.), la boisson rituelle symbolique relève d'une consommation réelle. L'invitation doit être acceptée et néanmoins dosée : nombreux sont ceux qui trempent les lèvres et assurent avoir avalé une rasade. Boire à 9 heures du matin, être le réceptacle de libation de rhum artisanal sur le haut du crâne, etc., sont autant d'éléments à gérer par les participants à un rituel (croyants ou ethnographe). De fait, ces obligations au partage ne se restreignent pas à la salle de soin et au temps rituel, mais peuvent, par exemple, accompagner le buveur ou le récipiendaire des libations jusqu'à un difficile retour olfactif en bus à travers la ville pour rejoindre son domicile ou son lieu de travail au sortir de la salle de soin du devin-guérisseur. Cet aspect est particulièrement remarquable lorsque l'odeur provient du sommet du crâne d'une ethnologue non malgache facilement repérable dans les transports en commun de la communauté urbaine de Fianarantsoa.

De ce fait, « méfions-nous de la vision angélique du boire fraternel abolissant toute différence. Partager la même bière crée des liens, mais aussi des obligations », remarque Eric Jolly dans le contexte malien (2003 : 109-110). Le boire rituel se répercute olfactivement et parfois gestuellement au-delà des murs de la salle de soins, 
dans l'espace public pour les buveurs. Le partage de boissons alcoolisées autorise les buveurs à accéder au rituel, mais les place également dans un rapport rituel hiérarchique (dominé par le guérisseur ou les esprits insistant sur la consommation et/ou les libations) qui impose des conduites. Par-là, l'alcool rituel est bon et puissant d'un point de vue magico-religieux, mais peut-être néfaste d'un point de vue corporel et social : " On a vu qu'un corps guéri est un corps propre, au contraire d'un corps alcoolisé qui est un corps souillé » (1995 : 114) analyse Sylvie Fainzang dans le contexte tout à fait éloigné du rapport à l'alcool des anciens buveurs français. Ce qui prévaut pour les anciens alcooliques, interlocuteurs de Sylvie Fainzang n'est pas adaptable à tous les guérisseurs malgaches. En effet, si RaJaona souille sa corporéité (diction et gestuelle), Sainte, quant à elle, sacralise la pratique rituelle à travers son corps alcoolisé. En ce sens, précisons encore à la suite d'Eric Jolly que « boire beaucoup ne signifie pas s'enivrer. La notion d'ivresse ou d'abus ne se fonde ni sur la quantité de bière ingurgitée ni sur le degré réel d'alcoolisation, impossible à mesurer. L'excès n'est perceptible qu'à travers la conduite négative ou anormale du buveur »(2004:107), et plus particulièrement, pour notre exemple, dans le cas rituel. Les participants aux rituels peuvent faire face à une consommation rituelle excessive temporaire par rapport à leur consommation habituelle, sans comparaison ni jugement avec les autres buveurs rituels. Comme pour RaJaona, « un homme ne boit trop qu'à partir du moment où il enfreint les normes du boire, d'une manière ou d'un autre. C'est là toute l'ambiguïté de la définition de l'ivresse, phénomène autant social que physiologique » (Ibid. : 122). Autrement dit, « mal boire ne se confond ni avec l'ivresse ni avec le trop boire » (Ibid.). En somme, « le discours sur l'alcoolique le situe toujours dans ses relations à autrui et au monde, et lorsque sont exprimés les désordres physiologiques associés à la maladie, c'est soit par rapport à l'autre, soit en référence à un comportement social » (Faizang 1995 : 123), telle que la sorcellerie pour notre exemple.

\section{Conclusion}

Pour conclure, les cas de Sainte et de RaJaona révèlent l'alcool sous la forme d'un Janus aux deux visages, comme le souligne Lionel Obadia, « il cristallise de concert un pathos qui en révèle les aspects morbides et asociaux, et un ethos, qui signale de son côté ses dimensions socialement et culturellement positives, comme ciment social ou figuration des valeurs culturelles d'un groupe humain » (2004 : 6). Les entités, les guérisseurs et parfois les participants associent tout autant qu'ils marquent une dissociation entre le sacré et le festif (Obadia 2009). Le guérisseur enivré ou l'esprit princier poursuivant le rituel en discussion potache et alcoolisée démontre comment le religieux glisse vers le festif et comment la fête se détache de la codification du rituel qui l'a amorcée.

A la suite de Claudine Fabre-Vassas, notons qu'il est nécessaire pour l'anthropologue de ne pas :

« perdre de vue la place cérémonielle qu'occupe le partage d'alcool dans bien des sociétés, loin de signifier la déviance, il réinstaure le lien social. Aussi doit-on prêter la plus grande attention non à la consommation d'alcool en soi, non à son abus défini dans l'absolu (il est tout relatif), mais à la position du buveur au sein de son groupe » (1989). 
Ainsi, même si RaJaona est considéré comme un alcoolique pathologique par certains de ses patients, il conserve son statut privilégié de consommateur d'alcool rituel efficace. Il est alors nécessaire de pondérer cette conclusion, et ainsi d'éviter une surinterprétation et une essentialisation de l'analyse des données en rappelant qu'une autre partie de ses patients estiment que la démesure de la consommation d'alcool de RaJaona menace l'efficacité du rituel. Néanmoins, le contexte rituel peut permettre une inversion des codes sociaux où la consommation d'alcool démesurée sans ébriété devient un outil de mesure des grandes compétences magico-religieuses d'un guérisseur. En somme, l'alcool trouve sa place dans le rituel et justifie son aspect magico-religieux mais peut également le conduire à l'échec. En effet, la démesure liée à la consommation d'alcool n'est autorisée que dans la limite de l'activité rituelle, dans la mesure rituelle. L'action rituelle est alors l'unique contexte légitime de son usage.

\section{Références citées}

Althabe, Gérard, 2002 [1969]. Oppression et libération : les communautés villageoises de la côte orientale de Madagascar. Paris : La Découverte.

Austnaberg, Hans, 2008. Shepherds and demons. A study of exorcism as practised and understood by shepherds in the Malagasy Lutheran church. New York: Peter Lang.

BEAujARD, Philippe, 1995. « Religion et société à Madagascar », in L'étranger intime. Mélanges offerts à Paul Ottino : Madagascar, Tahiti, Insulinde, Monde Swahili, Comores, Réunion, pp. 181-217. Saint Denis (Réunion) : Université de la Réunion : Diffusion Océan Indien.

BEAUJARD, Philippe, 2014. « Mythes et rituels. Le miel et l'hydromel dans quelques sociétés de Madagascar », Etudes océan Indien, n51-52, pp. 129-170.

Bianquis, Isabelle, 2012. L'alcool. Anthropologie d'un objet-frontière. Paris : L'Harmattan.

Blanchy, Sophie, 2006. "La vie comme un champ de forces : espaces et rites », in Sophie Blanchy, Jean-Aimé Rakotoarisoa, Philippe Beaujard et Chantal Radimilahy (dir.), Les dieux au service du peuple. Itinéraires religieux, médiations, syncrétisme à Madagascar, pp. 261-316. Paris : Karthala.

Blanchy, Sophie et Andriamampianina Rahajesy, 2001. «Possession, transe ou dialogue ? Les formes récentes de la communication avec les ancêtres en Imerina (Madagascar) », in Marie-Claude DuPRÉ (dir.), Familiarité avec les dieux, transe et possession (Afrique noire, Madagascar, La Réunion), pp.23-61. Clermont-Ferrand : Presses universitaires Blaise Pascal.

Blanchy, Sophie, Jean-Aimé Rakotoarisoa, Philippe Beaujard et Chantal Radimilahy (dir.), 2006. Les dieux au service du peuple. Itinéraires religieux, médiations syncrétisme à Madagascar. Paris : Karthala.

Bougerol, Christiane, 1993. « Le cumul magico-religieux à la Guadeloupe », Journal de la Société des Américanistes, 79, pp. 91-103.

CALlET, François, 1908. Tantara ny andriana. Tananarive : Imprimerie officielle.

Castelain, Jean-Pierre, 1996. « La quête des mots. De quelques usages de l'alcool dans la France de l'Ouest », Communications, 62, pp. 181-193.

Chemillier, Marc, Denis Jacquet, Victor Randriany et Marc Zabaia, 2007. « Aspects mathématiques et cognitifs de la divination sikidy à Madagascar », L'Homme, 181, pp. 7-37.

Douglas, Mary, 1987. Constructive Drinking. Perspectives on Drink from Anthropology. Cambridge : Cambridge University Press et Paris : Maison des sciences de l'homme.

Dubois, Henri 1938. Monographie des Betsileo. Paris : Institut d'ethnologie.

Estrade, Jean-Marie, 1985. Un culte de possession à Madagascar : le tromba. Paris : L'Harmattan.

FABre-VASSAS, Claudine, 1989. «La boisson des ethnologues », Terrain, 13, pp. 5-14.

FAINZANG, Sylvie, 1995. «L'alcool, les nerfs, le cerveau et le sang », l'Homme, 135, pp.109-125. 
FAUblÉE, Jacques, 1946. Ethnographie de Madagascar. Paris : Editions de France et d'Outre-Mer, La Nouvelle Edition.

FAVRET-SAADA, Jeanne, 2004 [1977]. Les mots, la mort, les sorts. Paris : Folio essais, Gallimard.

FIÉLOUX Michèle et Jacques LOMBARD, 1991. Le prince charmant, production IRD/MRSTD Madagascar, édition CERIMES (Centre de ressources et d'information sur les multimédias pour l'enseignement supérieur), $43 \mathrm{mn}$.

FIÉLOUX, Michèle et Jacques LOMBARD, 1995. «Du royaume à la ville : le territoire des possédés (Madagascar) », in Jeanne-Françoise VInCENT, Daniel Dory et Raymond Verdier (éds), La construction religieuse du territoire, pp. 323-337. Paris : L'Harmattan.

FiskesJö, Magnus, 2010. « Participant Intoxication and Self-Other Dynamics in the Wa context », The Asia Pacific Journal of Anthropology, 11 (2), pp. 111-127.

GIVRE, Olivier, 2006. Un rituel « balkanique » ou un rituel dans les Balkans? Approche anthropologique du kourban en Bulgarie et en Grèce du nord. Thèse de doctorat en anthropologie. Université Lyon 2.

Hell, Bertrand, 1991. « La force de la bière. Approche d'une récurrence symbolique dans les systèmes de représentations de l'Europe nord-occidentale », in Dominique FourniER et Salvatore D'ONOFrio (dir.), Le ferment divin, pp. 109-123. Paris : Editions de la Maison des sciences de l'homme.

HeLl, Bertrand, 2008. « Négocier avec les esprits tromba à Mayotte », Gradhiva, 7, pp. 6-23.

JoLly, Eric, 2003. " L'ivresse du terrain. Une enquête sur la bière de mil dans la société dogon (Mali) », in Lionel Obadia (dir), L'ethnographie comme dialogue. Immersion et interaction dans l'enquête de terrain, pp. 90-136. Paris : Publisud.

Jolly, Eric, 2004. Boire avec esprit. Bière de mil et société dogon. Nanterre : Société d'ethnologie.

LaCAZE, Gaelle, 2004. «Convivialité et consommation d'alcool chez les Mongols et les Kazakhs », Annales de la fondation Fyssen, 19, pp. 31-47.

Legrip-Randriambelo, Olivia, 2014. (Dé)loger le mal. Spatialité et pratiques religieuses de guérison en région betsileo (Madagascar). Thèse de doctorat en anthropologie. Université Lyon 2.

LÉvi-Strauss, Claude, 1964. Mythologiques. Le cru et le cuit. Paris : Plon.

Nahoum-Grappe, Véronique, 1989. “'Boire un coup...'”, Terrain, 13, pp. 72-80.

NiELSSEn, Hilde, 2012, Ritual imagination. A study of Tromba Possession among the Betsimisaraka in Eastern Madagascar. Leiden/Boston : Brill.

OBADIA, Lionel, 2004. « Le 'boire'. Une anthropologie en quête d'objet, un objet en quête d'anthropologie », Socio-anthropologie, 15, pp. 3-30.

OBADIA, Lionel, 2005. La sorcellerie. Paris : Le cavalier bleu.

OBADIA, Lionel, 2009. « Le petit bal qui finit mal... un invariant de la fête ? », in Laurent Sébastien FourNIER, Dominique Crozat, Catherine Bernie-Boissard et Claude Chastagner (dir.), La fête au présent. Mutations des fêtes au sein des loisirs, pp. 17-25. Paris : L'Harmattan.

Ottino, Paul, 1965. « Le tromba (Madagascar)», L’Homme, 5 (1), pp.84-93.

Petit, Pierre, 1997. «Vu des Antipodes : versions africaines de l'exotisme », Traces (Travaux du Centre d'études Georges Simenon), 9, pp. 179-192.

RAHAMEFY, Adolphe, 1997. Le roi ne meurt pas. Rites funéraires princiers du Betsileo. Madagascar. Paris : L'Harmattan.

Rakotomalala, Malanjaona, Sophie Blanchy, et Françoise Raison-Jourde (dir.), 2001. Madagascar: les ancêtres au quotidien. Usages sociaux du religieux sur les Hautes-Terres malgaches. Paris : L'Harmattan.

RIFFARD, Claire, 2008. «Les débuts de la poésie écrite en langue malgache », Etudes océan Indien, 40-41, pp. 81-100.

Sharp, Lesley A., 1993. The possessed and the dispossessed. Spirits, Identity, and Power in a Madagascar Migrant Town. Oakland (CA) : University of California Press.

Tarabout, Gilles, 1999. « Prologue. Approches anthropologiques de la possession en Asie du Sud », in Jackie Assayag et Gilles Tarabout (dir.), La possession en Asie du Sud : parole, corps, territoire, pp. 9-30. Paris : Editions de l'EHESS. 